\title{
MRI of the Brain in Moving Subjects Application to Fetal, Neonatal and Adult Brain
}

\author{
P. Narendran \\ Head \& Associate Professor, PG \& Research Department of Computer Science, \\ Gobi Arts \& Science College (Autonomous), \\ Gobichettipalayam - 638 453, Erode District, Tamil Nadu, India \\ Email ID: narendranp@gmail.com \\ V. K. Narendira Kumar \\ Assistant Professor, Department of Information Technology, \\ Gobi Arts \& Science College (Autonomous), \\ Gobichettipalayam - 638 453, Erode District, Tamil Nadu, India \\ Email ID: kumarmcagobi@yahoo.com \\ Dr. K. Somasundaram \\ Head \& Professor, Dept. of Computer Science \& Applications, \\ Gandhigram rural university, \\ Gandhigram - 624 302, Tamil Nadu, India \\ Email ID: somasundaramk@yahoo.com
}

\begin{abstract}
Imaging in the presence of subject motion has been an ongoing challenge for magnetic resonance imaging (MRI). In this paper some of the important is sues regarding the acquisition and reconstruction of anatomical and DTI imaging of moving subjects are addressed; methods to achieve high resolution and high Signal to Noise Ratio (SNR) volume data. Excellent fetal brain 3D Apparent Diffusion Coefficient maps in high resolution have been achieved for the first time as well as promising Fractional Anisotropy maps. Growth curves for the normally developing fetal brain have been devised by the quantification of cerebral and cerebellar volumes as well as someone dimensional measurements. A Verhulst model is to describe these growth curves, and this approach has achieved a correlation over 0.99 between the fitted model and actual data.
\end{abstract}

Index Terms-Brain, MRI, Registration, Fetal, Neonatal, Adult

\section{Introduction}

Magnetic Resonance Imag ing (MRI) has proved to be one of the most important and valuable imaging modalities that produce high quality clinical images in a noninvasive way. MRI of the central nervous system (CNS), especially the brain, is attracting the most interest both anatomically and functionally. Despite many well established MR techniques targeting various pathologies, technical obstacles remain in certain situations, for example, imaging subjects under motion.
During standard MR scanning, especially brain examinations, the patient are normally required to keep still. However, this is onerous for patients with specific diseases, e.g., dementia, especially when scanned with sequences that require several minutes' acquisition. Moreover, it is also very difficult for the pediatric patients to keep still without sedation. Even with sedation, those patients especially the neonates may be disturbed or woken up by noisy scanning sequences [2]. Most specifically, in-utero fetal MR imaging is very vulnerable to both the unintentional motion of the fetus itself and the mother's respiration. Therefore, although highly desirable, there is currently no imaging technique from any imaging modality that can achieve high resolution volumetric imaging of the fetal brain. Besides anatomical MR imaging, motion will also cause severe problems to functional imaging. For instance, motion can invalidate diffusion tensor estimation in Diffusion Tensor Imaging (DTI). Motion will also bring error in functional MRI (fMRI) parameter, especially when there is intra-volume motion [8].

An approach to acquisition and reconstruction of anatomical and DTI MR imaging suitable for use on moving subjects is developed; methods to achieve high resolution and high SNR volume data are proposed. They have been applied successfully for brain studies on subjects that cannot stay still; and in some cases were moving substantially during scanning. For example, awake neonates, confused or distressed adults and, especially fetuses, for which no conventional high resolution $3 \mathrm{D}$ method is currently available. 


\subsection{Background Theory of MRI}

Magnetic resonance imaging is of immense use in clinical practice for producing anatomical and functional images to aid diagnosis as a powerful noninvasive medical imaging technique. Bloch and Purcell discovered the nuclear magnetic resonance phenomenon independently in 1946. Its use in image formation was not demonstrated until 1973 but by the early 1980s diagnostically useful images were being produced. Introduce the basic principles of magnetic resonance imaging which can be found in a number of texts. Then, a number of sequences, which have been used to acquire data for various facets of this work, are detailed [4].

\subsection{Existing Challenges}

MRI has been widely adopted clinically as a crucial supplement for ultrasound in in-utero fetal examination especially for pathologic diagnosis. Techniques have been developed since 1983 to allow the early development of human brain to be studied in superior detail as described in previous sections. However, many challenges remain [5].

Anatomical Imaging: The unpredictable fetal motion has led to widespread use of single shot techniques, e.g. the ssTSE sequence, to freeze fetal motion and get a series of high quality 2D slices. However, in a conventional clinical exam, resolution and slice thickness are generally compromised to maintain signal-to-noise ratio (SNR). In addition, although parallel slices are prescribed in the scanner coordinates, the anatomical brain slices obtained are frequently inconsistent because of changes in fetal position between shots. It is therefore generally not possible to combine multiple slices into a self consistent view of the 3D brain.

Functional Imaging: An even greater challenge is in-utero Diffusion Weighted Imaging (DWI) and Diffusion Tensor Imaging (DTI). This is mainly due to the technical barrier in the conventional diffusion weighted sequence that is highly sensitive to fetal motion and maternal respiration. Motion not only produces various artifacts in the diffusion weighted images, but also invalidates the evaluation of the diffusion tens or matrix, and of course, the ADC and FA values. Therefore, to increase the SNR, a large voxel size has to be used. However, high resolution because of the tiny size of fetal brain is very desirable to achieve better localization of the response [1].

\section{Methodol ogy}

In this paper, an approach to acquisition and reconstruction of anatomical and DTI MR imaging suitable for use on moving subjects is developed; methods to achieve high resolution and high SNR volume data are proposed. They have been applied successfully for brain studies on subjects that cannot stay still; and in some cases were moving substantially during scanning. For example, awake neonates, confused or distressed adults and, especially fetuses, for which no conventional high resolution $3 \mathrm{D}$ method is currently available.

\subsection{Acquisition Details}

For ex-utero scanning, including neonatal, pediatric and adult scanning, three multi slice sequence variations with either T2 weighted or dual echo achieving both T2 and Proton Density weighted imaging were set up on a 3.0 T Philips Achieva scanner using an 8 channel SENSE head coil

\subsection{Subjects}

This method has been tested on 11 neonatal subjects with multi slice T2 sequence as well as 6 adult data from the IXI cohort of normal volunteers with a dual echo sequence. Motion artifacts like spatial inconsistency in the through slice direction and aliasing were quite obvious in the original acquired image. For ex-utero scans, segmenting the brain from non-brain tissue is not needed, therefore, this is a fully automatic process.

\subsection{Registration and Reconstruction Algorithm}

The multi-slice data was separated into $\mathrm{N}$ separately acquired packages containing spatially separated 2D slices and each package was treated as an independent set of samples of the same rigid body object. One package that is most consistent and has least motion artifact is selected as the target for co-registering each package together at first. All the gap slices in each package are interpolated using cubic B-spline interpolation, thus making $\mathrm{N}$ synthesized volumes of the subject imaged under different spatial orientations [8].

Volume-to-volume rigid body registration using gradient decent as the optimization method was implemented here to co-register these synthetic volumes. Then, these packages of interleaved slices but not these synthetic volume were then reconstructed together into a single volume using a scattered data interpolation algorithm based on multilevel b-spline interpolation with control point spacing in the through slice direction set to equal the slice gap in a single package.

This results in a mean brain volume image that can be used as a registration target for each package. In all cases final reconstruction uses control points spaced at half the acquired resolution both in plane and through slice as this has been found to provide an effective balance of resolution and signal to noise ratio [6].

\subsection{Subjects and Scanning protocols}

Fetal brain images were acquired on a $1.5 \mathrm{~T}$ Philips Achieva scanner (Best, The Netherlands) using a 5 
channel torso array with a spin echo planar diffusion tensor sequence. The fetal brain images were preprocessed to isolate the region containing the head from the womb using a Semi-automated segmentation method.

We first segment one loop of the fetal brain by roughly tracing the edge of the head manually to exclude maternal tissues. For a fetal data set consisting of 72 slices, the whole process takes around 20 minutes using Image. The fetal brain is then extracted from all the other images using registration based segmentation. This segmentation is later refined by interleaving it with the registration process used to determine slice alignment, allowing both registration and segmentation to be iteratively improved.

All slices were inspected and the slices that were corrupted by in-plane motion were excluded before registration and reconstruction. A high resolution anatomical volume acquired with $\mathrm{T} 2-\mathrm{W}$ single short Turbo Spin Echo (ssTSE) dynamic sequence as described in SVR was also reconstructed for comparison.

\subsection{SVR Method}

To achieve high resolution 3D brain images of the fetal brain we have developed a complementary approach that uses dynamic single shot scanning to sample the spatial region containing the brain with multiple overlapping slices. Image registration is then used to retrospectively align the images obtained based on the assumption that they represent a single rigid body undergoing an unknown motion. Having determined the correct location of each image in a self consistent anatomical space of the fetal brain, we regard the measured voxel intensities fro $m$ all the slices as valid samples at known although irregularly spaced locations and use a scattered interpolation approach to reconstruct an optimal estimate of the 3D fetal brain [1]

We term this concept of using dynamic single shot slice by slice imaging combined with retrospective align ment and data fusion to produce self consistent 3D volume images Snapshot MRI with Volume Reconstruction or SVR. Although inspired by fetal scanning it has wide application to imaging moving objects that can be assumed to have a constant shape, particularly when there may be other objects in the field of view that undergo differential motion and/or changes in shape. We have therefore tested the method in the general context of imaging the brains of subjects who cannot remain sufficiently still for conventional MRI examinations.

\section{Implementation of the MRI System}

Fetal brain images were acquired on a Philips $1.5 \mathrm{~T}$ Intera system. Test data and images of the brains of neonates, children and adults were acquired at $3 \mathrm{~T}$. The basic sequence uses ssTSE methods with half Fourier data collection to acquire multiple T2 weighted 2D slices. The imaging was performed as dynamic loops with the scanner operating continuously to acquire parallel slices at a rate of about $1 /$ second. The scans were specified as loops of continuous and overlapping slices with slice thickness $2 \mathrm{H}$ and slice gap $-\mathrm{H}$ to allow slice separation $\mathrm{H}$ and achieve dense sampling as described before. Slices were acquired in 4 packages and within each packages starting at one extre me end in an interleaved order, For a slice acquisition rate of $\sim 1 /$ sec with 60 slices covering the region of interest the total loop time would be 60 seconds and for each acquired slice the closest slices were excited at least 15 seconds previously or subsequently so that the relaxation history in the vicinity of the current slice is effectively uniform.

\subsection{Registration Algorithm}

The method requires individual 2D slices to be registered to a $3 \mathrm{D}$ volume. This is a difficult problem, particularly in the premature brain which has less complex patterns of sulcation than adult brains. The rigid body transformations are specified by 6 parameters: 3 rotations and 3 translations; therefore, the optimization takes place in high dimensional space: R6. The problem present here is actually a global optimization. Therefore, the method used should ideally be tuned for the particular problem at hand - in this case, slice to volume registration using fetal brain MR images [8].

For registration of the adult and neonatal data, the same algorithm was used with different rotation ranges for each stage, since the head motion generally covers a smaller range of translations and rotations than for the fetus. Therefore, after initial volume-to-volume registration, a narrower range of rotations was adopted (e.g. -9 to 9 degree or -6 to 6 degree), and some stages with intermediate nu mbers of slices could be skipped to save computational time. If severe motion does occur, strictly following the fetal registration scheme provides excellent results.

\subsection{Test Data for Registration and Reconstruction Accuracy}

In order to test the accuracy of slice-to-volume registration, we first used a set of adult MR images scanned with a T2-W ss TSE protocol using a T/R head coil. Each of the individual slices was registered to the target volume using slice-to-volume registration. To test the reconstruction accuracy a set of scattered data samples was created by sampling the gold standard object at random positions in all three dimensions. The number of samples was 8 times the number of gold standard voxels and the maximum sample separation was equal to the gold standard voxel size, so the data was both over-sampled and dense [6]. This scattered data was then reconstructed onto a regular grid using the multilevel scattered data approximation and compared with the gold standard. For both the 
registration and reconstruction tests noise was added to the gold standard data to recreate SNR levels similar as that of the actual fetal scans at $1.5 \mathrm{~T}$. To cover the entire neonatal brain required 72 slices for each loop in the transverse orientation, 80 slices for each loop in the coronal orientation and 64 slices for each loop in the sagittal orientation to be synthesized [8].

\subsection{SNR Test}

The effect of data compounding and of the reconstruction on SNR and resolution were also tested with the neonatal dataset. Four copies of the data were created with regular samples at unit intervals, but shifted progressively by $1 / 4$ of a slice thickness in the slice direction and with independent Gaussian noise added [8]. Another numerical experiment was performed by adding independent Gaussian noise with increasing STD to the synthesized motion corrupted neonatal dataset to create data sets with SNR 11, 8.3 and 6.7 corresponding to SNR values for slice thicknesses of $3.3 \mathrm{~mm}, 2.5 \mathrm{~mm}$ and $2 \mathrm{~mm}$ respectively at typical $1.5 \mathrm{~T}$ performance. These data were then reconstructed by SVR and compared to the original data [7]. Finally motion corrupted fetal, neonatal and adult data sets were each reconstructed by SVR using a final lattice control point spacing of $0.5 \mathrm{~L}(\mathrm{~L}=$ slice spacing in the scanner frame). The reconstruction programs were written in $\mathrm{C}++$ and processing was done on a $1 \mathrm{GHz}$ Pentium PC.

\section{Experimental Results}

Tests of the registration accuracy using synthesized motion corrupted data consisting of 4 loops in a transverse orientation with each slice undergoing a different rigid motion showed that all 249 slices were positioned accurately with cross correlation values above 0.93 after the final reg istration step. We found that these results were robust to the SNR of the individual images and the noise in the reconstructed image was almost Gaussian as well. SVR reconstruction also preserved the original high resolution of the source slice data, while making the whole volume consistent in space as well as increasing the SNR. Two profiles at corresponding edges of both reconstructed volume and original slice are shown in Figure $1(\mathrm{j}-\mathrm{k})$ allowing direct visual comparison. This qualitative test confirms that edge sharpness in anatomy is well preserved as well.

$>$ Figure 1 (a)-(c) are acquired fetal MR transverse data viewed in Transverse, Coronal and Sagittal planes with $1.25 \mathrm{~mm} .1 .25 \mathrm{~mm}$ in-plane resolution and $2.8 \mathrm{~mm} \mathrm{TH}$.

$>$ Figure 1 (d)-(f) are the corresponding views for averaged images from the full dynamic scan before motion correction.
$>$ Figure 1 (g)-(i) are the corresponding views reconstructed to $1.25 \mathrm{~mm}$ isotropic resolution image after registration.

$>$ Figure 1 (j)-(k) are measured edge profiles of original acquired slice (marked as white line in (a)) and corresponding position at reconstructed image (marked as white line in $(\mathrm{g})$ ).

$>$ Figure 1 (1)-(n) are the corresponding views of a preterm neonate born at 28 weeks and scanned at 34 week with $0.98 .0 .98 \mathrm{~mm}$ in-plane resolution and $1 \mathrm{~mm}$ TH.
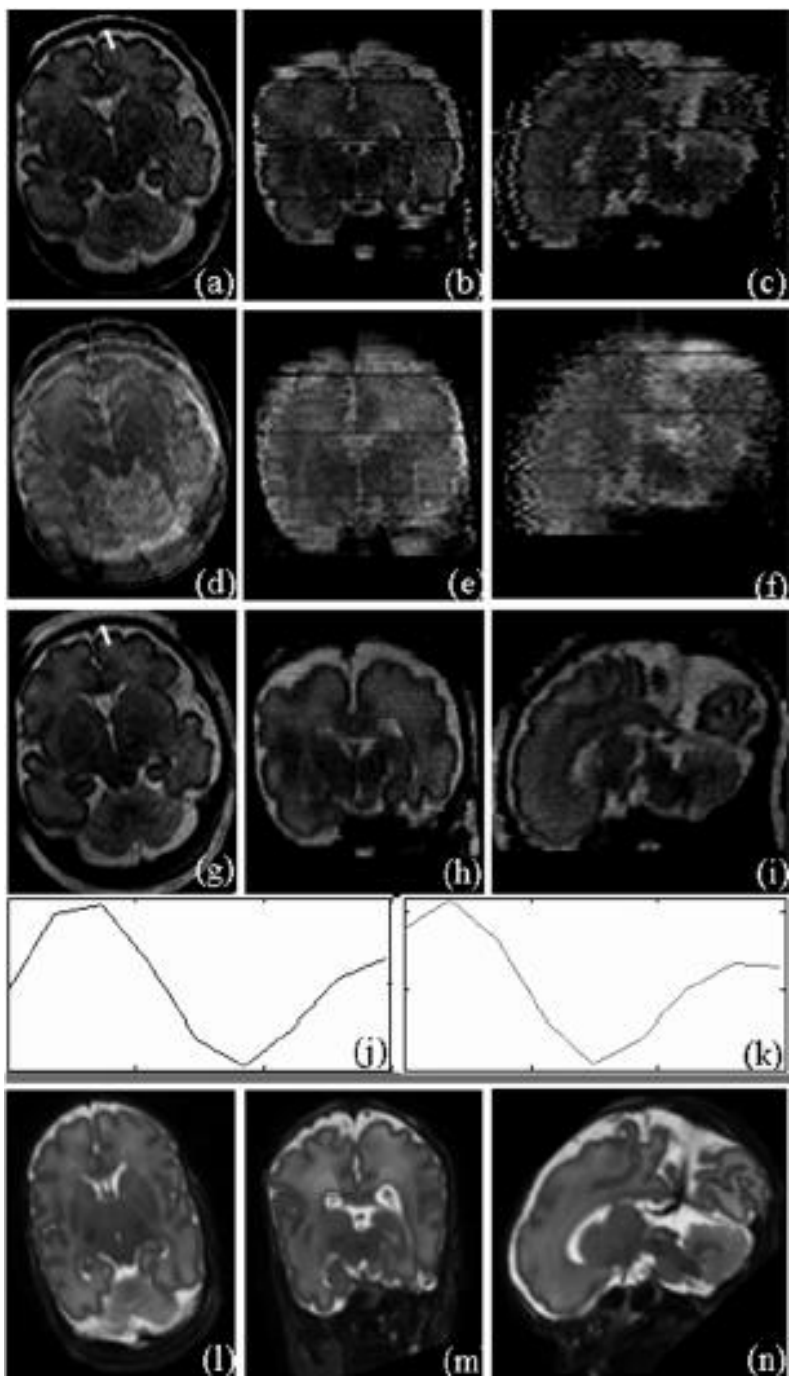

(k)

Fig. 1: Result for a normal fetus of 33 week gestational age

However, the $3 \mathrm{~T}$ acquired data on adult brain we found that changing slice orientation resulted in differences in the data that introduced errors larger than the registration uncertainties. We interpret this result as evidence of slice distortion caused by changing interactions between the image encoding gradients and background susceptibility effects caused by the subject being in the scanner. In the adult brain data we collected we did not find this a significant problem at the lower field like 1.5T. We did not detect any slice distortion effects in the fetal data acquired, however 
since there is no motion free reference in this case we cannot rule this out.

\subsection{Fetal Results}

All twenty fetal datasets were successfully reconstructed. Two examples are illustrated here to display reconstructed results with different acquired slice thickness and with only parallel slices or slices from all three orthogonal orientations. Figure 1 shows data from a fetus of 33 weeks gestation scanned using 4 dynamic loops of $60.2 .5 \mathrm{~mm}$ slices prescribed as nominally in the transverse orientation. Figure 2 shows data from a less mature fetus of 28 gestation weeks combining 4-loops of transverse, 2-loops of coronal and 2-loops of sagittal slices with slice thickness $2.5 \mathrm{~mm}$ and $1.25 \mathrm{~mm}$ slice separation. In these figures, the top row shows a single loop of the orig inal fetal image data in a 3-plane view (transverse, coronal and sagittal) [3]. Although the native (transverse) plane is as expected, the other views reveal motion corruption. Simply combining all the acquired slices without correcting for fetal motion resulted in blurred images (second row in Figure 1 - Figure 2).
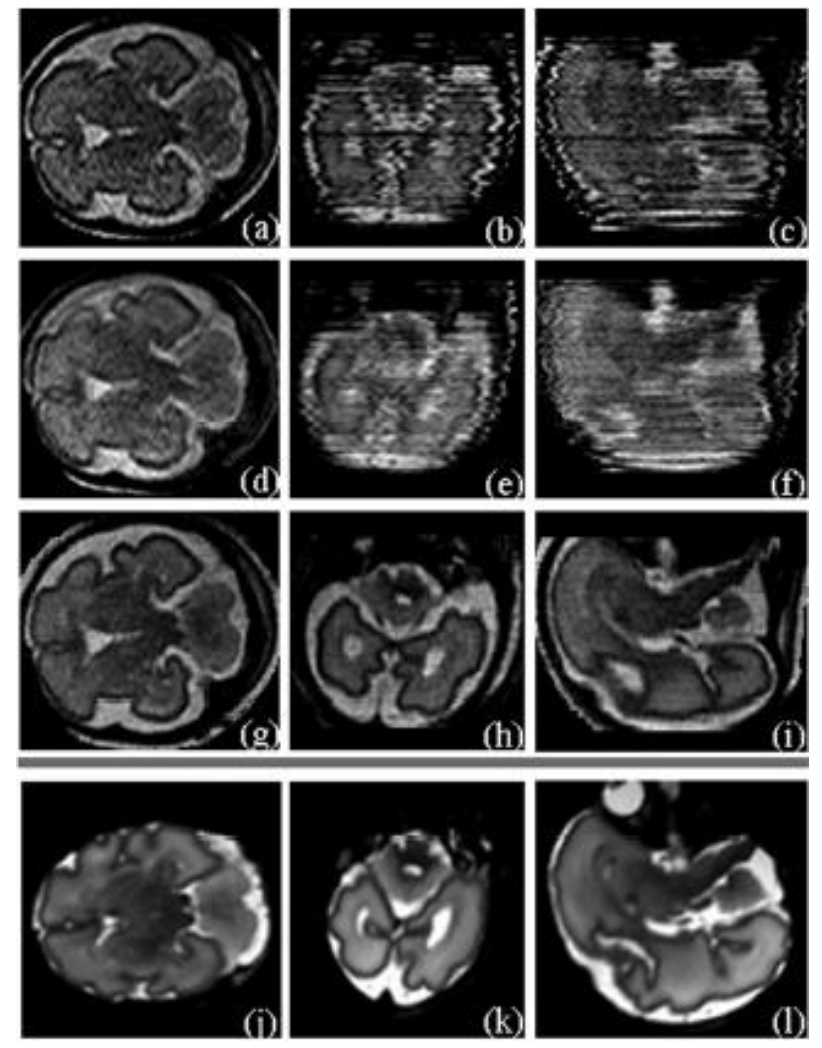

Fig. 2: Result for a normal fetus of 28 week gestational age

$>$ Figure 2 (a)-(c) are acquired fetal MR transverse data viewed in Transverse, Coronal and Sagittal planes with $1.25 \mathrm{~mm} .1 .25 \mathrm{~mm}$ in-plane resolution and $2.5 \mathrm{~mm} \mathrm{TH}$.

$>$ Figure 2 (d)- (f) are the corresponding views for averaged images from the full dynamic scan before motion correction.
$>$ Figure 2 (g)- (i) are the corresponding views reconstructed to $1.25 \mathrm{~mm}$ isotropic resolution image after registering slices from 3 orthogonal orientations.

$>$ Figure 2 (j)- (1) are the corresponding views of a preterm neonate born at 28 week and scanned at 30 week with $0.98 .0 .98 \mathrm{~mm}$ in-plane resolution and 1 mm TH.

At the final stage of image registration, Data 1 (Figure 1) uses 208 slices and Data2 (Figure 2) uses 327 slices. In each case a $1.25 \mathrm{~mm}$ isotropic reconstruction of a 3D self-consistent volume with improved SNR and high contrast between cortical grey and white matter is produced. Details of cortical folding and small structures in the cerebella are well displayed in all three planes (third row in Figure 1- Figure 2). Additionally, when compared to preterm born infants scanned at similar gestational age but at 3 Tesla (last row in Figure 1 - Figure 2), strong correlations in brain anatomy including cortical folding, deep white matter tracts and cerebellar structures can be found [2].

\subsection{Adult Results}

This method can also be applied to other brain studies in both children and adults, wherever motion is a problem. This has been first tried on an adult volunteer study in which the subject deliberately moved about once per second, including making head rotations of up to 15. For comparis on, images were also acquired with the same protocol while the subject was still. Because of this intense motion, some slice images were found to be degraded by movement during the single shot it. All the slices that were not corrupted by in plane motion were accurately registered and used for reconstructing a 1.0.1.0.1.4 $\mathrm{mm}$ volume, which is almost identical to the motion free image with doubled SNR as displayed in Figure 3. Thus even in this extreme case, the method is able to recover detailed artifact free images.

$>$ Figure 3 (a)-(c) are acquired MR transverse data viewed in Transverse, Coronal and Sagittal planes with $1.0 \mathrm{~mm} .1 .0 \mathrm{~mm}$ in-plane resolution and 2.8 $\mathrm{mm}$ TH.

$>$ Figure 3 (d)-(f) are the corresponding views reconstructed to a 1.0.1.0.1.4 $\mathrm{mm}$ resolution image after registration.

$>$ Figure 3 (g)-(i) are the corresponding views of the same volunteer scanned with the same protocol but stayed still. Reduced coverage is realized because of limitation of scanning time.

The reconstruction has successfully achieved 3D coherent volume with both high resolution and almost doubled SNR despite of totally motion corrupted input data [6]. 

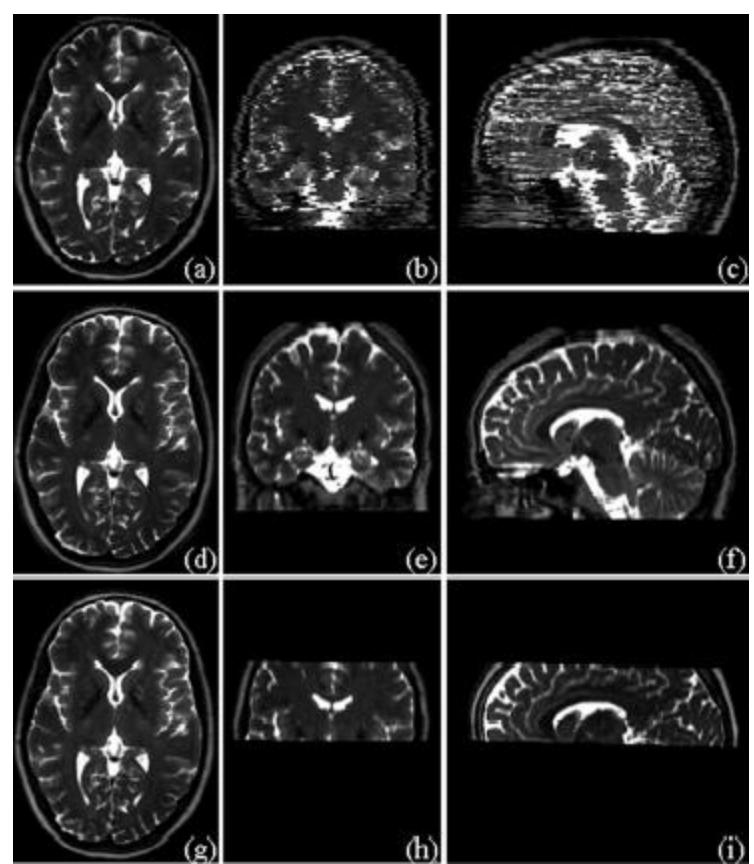

Fig. 3: Results for Adult volunteer that deliberately moved during scanning

\subsection{Neonatal Results}

A general application of SVR can be found in pediatric MR scanning including both neonates, who may move during natural sleep or sedation and young children who have difficulty staying still during scanning. By prescribing the slices as overlapped in their native planes, there is full sampling even if the subject does not move. This method can correct motion for each $2 \mathrm{D}$ slice and then achieve 3D coherent imaging with $1 \mathrm{~mm}$ isotropic resolution and almost doubled SNR.
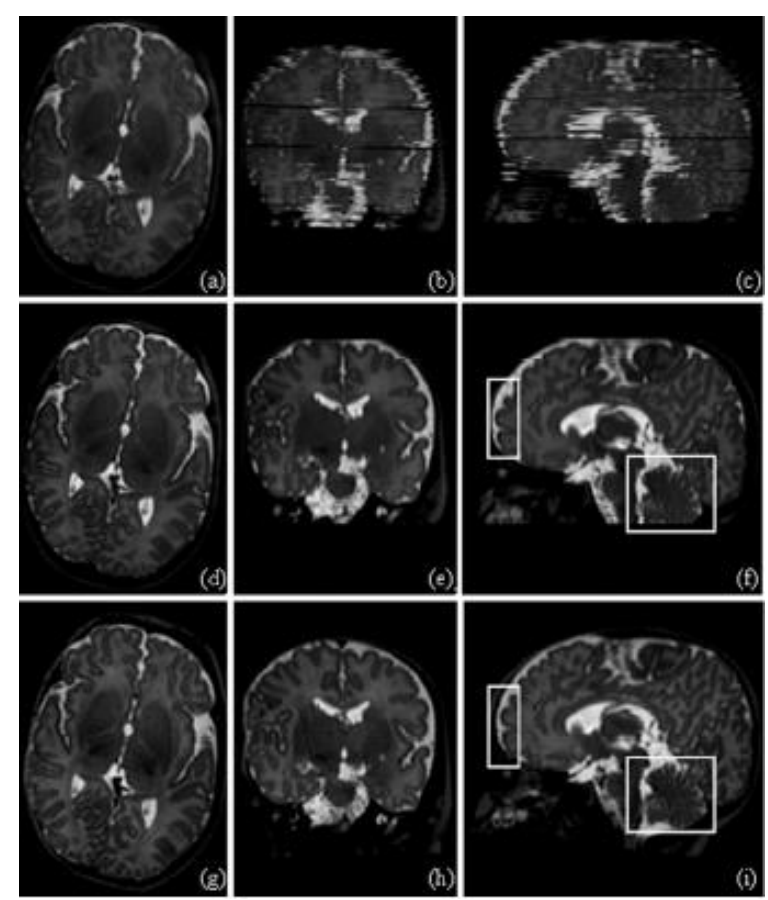

We scanned at $3 \mathrm{~T}$ with the top-bottom direction as the slice select direction.

$>$ Figure 4 (a) Shows one loop of ssTSE dynamic scan when the neonates is moving.

$>$ Figure 4 (b) Shows the reconstruction with 4 loops of ssTSE dynamic scan that achieved both coherent imaging and improved SNR.

$>$ Figure 4 (c) Show an acquisition with a TSE sequence with subject being still. Although the subject was sedated, some motion artifacts that make structures slightly in-consistent are still visible as highlighted in the rectangle regions (basal ganglia, cerebella, and frontal cortex) in Figure 4 (i).

$>$ The result compares well with the conventional multi-shot images acquired before the baby woke up. It is a high-fidelity reconstruction that is nearly identical when compared to actually required image when it was almost still (Figure 4, bottom row).

\section{Conclusion}

We have described a novel methodology, SVR, for imaging moving subject at high resolution and high SNR by combining reg istered $2 \mathrm{D}$ s lices from sequential dynamic single-shot scans. The SVR method requires that the anatomy in question is not changing shape or size and is moving at a rate that allows snapshot images to be acquired. It has been performed successfully on neonates, adults and especially on fetuses, for which no conventional high resolution $3 \mathrm{D}$ method is currently available. Fine structure of the in-utero fetal brain is clearly revealed for the first time and substantial SNR improvement is realized by having many individually acquired slices contribute to each voxel in the reconstructed image. The price of the benefits offered by this technique is increased scan time to allow multiple loops to be acquired. The ability to acquire data with certainty that it will yield artifact free images allows a reduction in set up time and in repeated examinations that can in fact increase examination efficiency. For adult and pediatric subjects the method offers a capability to obtain high quality volume data even when the subject is unable to cooperate with the examination and may be moving by many millimeters or turning by many degrees during the acquisition period. Validation using both adult and neonate simulated data has been performed to verify that the results obtained are consistent with the known ground truth and the reconstruction is found to be faithful.

\section{References}

[1] Adamsbaum,C., (2011). - Myelin characterization of fetal brain with mono-point estimated T1-maps. Magn Reson. Imaging 22, 565-572.

Fig. 4: Results for a neonate of 43 week GA who was unsettled in light sleep and moving during the scanning 
[2] Bydder,G.M. (2001). Diffusion-weighted imaging of the brain in neonates and infants. Magn Reson. Imaging Clin. 83-98,

[3] Chang,C.H., (2000). Assessment of fetal cerebellar volume using three-dimensional ultras ound. Ultrasound Med. Biol. 981-988.

[4] Chen,Q. (2001). Fast fetal magnetic resonance imaging techniques. Top. Magn Reson. Imaging 12, 67-79.

[5] Sands,J. (2011). Quantitative growth and development of human brain. Arch. Dis. Child 48, 757-767.

[6] Glover,G.H (2002). Projection reconstruction techniques for reduction of motion effects in MRI. Magn Reson. Med. 28, 275-289.

[7] Grossman,R., (2006). Quantitative MRI measurements of human fetal brain development in utero. Neuroimage. 463-470.

[8] Jenkinson,M., (2002). Improved optimization for the robust and accurate linear registration and motion correction of brain images. Neuroimage. 17, 825-841.

\section{Authors' Profile:}

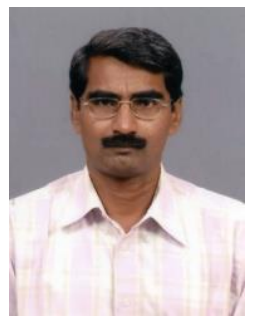

Mr. P.NARENDRAN M.Sc., PGDCA, M.Phil., B.Ed., is the Head \& Associate Professor, Department of Computer Science, Gobi Arts \& Science College (Autonomous), Gobichettipalayam - 638 453, Erode District, Tamil $\mathrm{Nadu}$, India. He received his $\mathrm{M}$. Phil., degree in Computer Science in 1995. His research interests include medical image processing.

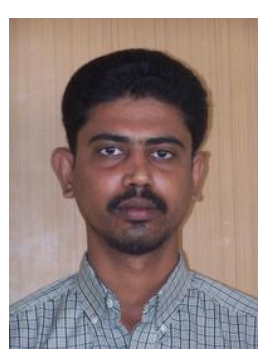

\section{Mr. V.K. NARENDIRA} KUMAR M.C.A., M.Phil., Assistant Professor, Department of Information Technology, Gobi Arts \& Science College (Autonomous), Gobichettipalayam - 638 453, Erode District, Tamil $\mathrm{Nadu}$, India. He received his M.Phil Degree in Computer Science from Bharathiar University in 2007. He has authored or co-authored more than 38 technical papers and conference presentations. He is an editorial board me mber and reviewer for several scientific international journals. His research interests are focused on advanced network, image processing, video processing, visual human-computer interaction, and multiple biometrics technologies.

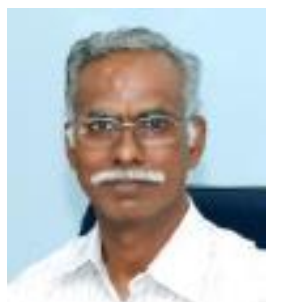

Dr. K. SOMAS UNDARAM, is Head \& Professor, Department of Computer Science \& Applications, Gandhigram Rural University, Gandhigram - 624 302, Tamil Nadu, India. He received his Ph. D. in the year 1984 from Indian Institute of Science, Bangalore. He has published 32 papers and 3 books. His research interests include Medical Imag ing, Image Compression, Image Enhancement, Multimedia for Teaching, PCBased instrumentation and Theoretical and Computational Plasma Physics.

How to cite this paper: P. Narendran, V. K. Narendira Kumar, K. Somasundaram,"MRI of the Brain in Moving Subjects Application to Fetal, Neonatal and Adult Brain", International Journal of Information Technology and Computer Science(IJITCS), vol.4, no.10, pp.44-50, 2012. DOI: $10.5815 /$ ijitcs.2012.10.05 